\title{
Phénoménologie clinique du corps propre
}

\section{Clinical Phenomenology of the one's own body}

\section{Fenomenologia clínica do corpo próprio}

\author{
Virgínia Moreira ${ }^{1}$ \\ Lucas Bloc ${ }^{2}$
}

\section{Résumé}

Inspirée par la phénoménologie philosophique, la phénoménologie clinique nous permet de prendre en considération la construction du discours théorique de la psychopathologie à partir du contact clinique. Dans cet article, nous avons comme objectif explorer les contributions de Merleau-Ponty et Tatossian pour la constitution d'une phénoménologie clinique du corps propre. L'article est structuré en trois axes : 1) la relation d'implication et non d'application entre les phénoménologies philosophique et clinique ; 2) la notion de corps propre chez Merleau-Ponty ; 3) l'idée du corps que je suis versus celui que j'ai dans la phénoménologie clinique de Tatossian pour comprendre le vécu dépressif. À travers la notion de corps propre, la phénoménologie clinique est susceptible d'avoir une contribution importante dans le développement de traitements et d'interventions qui comprennent de façon ambiguë le corps comme sujet et objet, touchant et touché, le corps que je ne peux être que si je l'ai.

Mots clés: Phénoménologie clinique; Corps propre; Merleau-Ponty; Tatossian; Dépression

\begin{abstract}
Inspired by philosophical phenomenology, clinical phenomenology allows us to take into consideration the construction of psychopathology's theoretical discourse based on clinical contact. In this article, we aim to explore the contributions of Merleau-Ponty and Tatossian for the establishment of a clinical phenomenology of one's body. The article is structured in three strands: 1) the relation of implication, rather than of application, between philosophical and clinical phenomenology; 2) Merleau-Ponty's one's own body notion; 3) the idea of the body I am versus the body I have in Tatossian's clinical phenomenology, which will bring out an understanding of the lived body in depression. Through the one's own body notion, clinical phenomenology is more likely to produce an important contribution to the
\end{abstract}


development of treatments and interventions that understand the body in an ambiguous way as a subject and object, as touching and touched at the same time; the body I can only be as long as I have it.

Keywords: Clinical phenomenology; One's own body; Merleau-Ponty; Tatossian; Depression

\section{Resumo}

Inspirada pela fenomenologia filosófica, a fenomenologia clínica nos permite levar em consideração a construção do discurso teórico da psicopatologia a partir do contato clínico. Neste artigo, temos como objetivo explorar as contribuições de Merleau-Ponty e Tatossian para a constituição de uma fenomenologia do corpo próprio. O artigo está estruturado em três eixos: 1) a relação de implicação e não de aplicação entre as fenomenologias filosófica e clínica; 2) a noção de corpo próprio em Merleau-Ponty; 3) a ideia do corpo que sou versus o corpo que tenho, na fenomenologia clínica de Tatossian, para compreender o vivido depressivo. Através da noção de corpo próprio, a fenomenologia clínica fornece uma contribuição importante no desenvolvimento de tratamentos e de intervenções que compreendem de forma ambígua o corpo como sujeito e objeto, tocante e tocado, o corpo que eu só posso ser se eu o tenho.

Palavras-chave: Fenomenologia clínica; Corpo próprio; Merleau-Ponty; Tatossian; Depressão

\footnotetext{
${ }^{1}$ Universidade de Fortaleza - UNIFOR - Fortaleza (CE), Brazil. E-mail: virginiamoreira@ unifor.br

${ }^{2}$ Universidade de Fortaleza - UNIFOR - Fortaleza (CE), Brazil; Université Paris Diderot - Paris VII. CAPES (Proc. 0998-14/1).
}

Cet article fait partie du projet $\mathrm{n}^{\circ} 1965$, appel d'offre 05/2016, aide supplémentaire pour la recherche à l'Université de Fortaleza - UNIFOR - Fortaleza (CE), Brazil.

Received: $1 / 28 / 2017$

Accept: $3 / 1 / 2017$ 


\section{Introduction}

Dès Platon et Aristote (Roux, 2010) le corps est un objet d'intérêt dans la philosophie et de plus en plus se présente comme un objet d'intérêt pour la clinique psychologique et psychiatrique. En ce qui concerne à la phénoménologie, en tant que courant philosophique, le corps est considéré comme une construction très importante pour la compréhension de l'être dans le monde, car c'est à travers lui que nous établissons des contacts avec le monde (Rovaletti, 1984). En mettant l'accent sur l'expérience du corps, Husserl et Merleau-Ponty, avec ses particularités, occupent une place centrale dans cette discussion et ont thématisé le corps dans son caractère propre. On peut dire que l'étude du corps vécu, au niveau phénoménologique, signifie prendre contact avec l'expérience de l'autre, évoquant le sens du monde ou de l'histoire de ce phénomène à l'état naissant (Merleau-Ponty, 1992/1945), c'est-àdire à travers ce qui est le plus propre et inaliénable - le corps (Bloc, Melo, Leite \& Moreira, 2015).

Dans la construction de ce qu'on appelle une phénoménologie clinique du corps propre, nous utilisons ici comme point d'ancrage philosophique la phénoménologie de Merleau-Ponty, lequel est pourtant peu cité lorsqu'il s'agit des propositions à ce niveau. Aussi bien que Husserl et Heidegger, référents majeurs, il peut se présenter comme interlocuteur (Bimbenet, 2011) et source d'inspiration. Sans aborder son long parcours et sa contribution à l'expérience du corps, l'un des thèmes majeurs de sa pensée (Angelino, 2008), nous utiliserons ses textes en tant qu'inspiration pour la clinique, ici comprise dans un sens large, s'appliquant à des modèles de clinique psychiatrique ou psychologique, mais aussi aux modèles de psychothérapie de nature phénoménologique. Bref, la phénoménologie de Merleau-Ponty sert d'inspiration pour une phénoménologie clinique en s'alliant avec d'autres auteurs de ce domaine.

La notion de corps n'est pas statique chez Merleau-Ponty, elle change tout au long de son œuvre. La notion de corps propre, qui apparaît plus concrètement dans la Phénoménologie de la Perception (1992/1945), se développe comme inter-corporéité et schéma corporel et se place comme Chair dans Le visible et l'invisible (1964) et aussi dans d'autres textes de la phase finale de sa pensée, de caractère plus ontologique.

Au niveau de la phénoménologie clinique, le corps a été évoqué comme un outil pour comprendre le vécu psychopathologique dès son début par des auteurs comme Binswanger, Minkowski, Tellenbach, et plus récemment par Tatossian, ce dernier en traitant d'une manière 
plus directe de la question de la clinique et de la psychopathologie et inspiré par une phénoménologie du corps propre. Tatossian doit être considéré comme un représentant contemporain de la phénoménologie clinique. Ses études sont marquées par la poursuite de l'appréhension de l'expérience psychopathologique authentique, qui consiste à comprendre le vécu comme phénomène doué de sens, ce qui va bien au-delà de la description simplement symptomatique.

Dans cet article, nous explorons les contributions de Merleau-Ponty et de Tatossian pour la constitution d'une phénoménologie clinique du corps propre et en tant qu'inspiration pour la prise du corps comme paradigme clinique. Les points suivants sont abordés : 1) la relation d'implication et non d'application, entre les phénoménologies philosophique et clinique ; 2) la notion de corps propre au début de la phase existentielle de la phénoménologie philosophique de Merleau-Ponty ; 3) l'idée du corps que je suis, versus celui que j'ai dans la phénoménologie clinique d'Arthur Tatossian, inspirée par la notion de corps propre, permettant de comprendre le vécu dépressif et d'illustrer l'apport à la clinique du corps propre.

\section{Phénoménologies philosophique et clinique}

L'utilisation de la phénoménologie dans le domaine de la clinique - la phénoménologie clinique - qui comprend la psychopathologie, la psychothérapie, la psychologie clinique et la psychiatrie - arrive plus tardivement et il est important de la différencier du domaine de la philosophie, même si elle s'en inspire (Moreira \& Bloc, 2015 ; Tatossian, 2002/1979; Tatossian et al., 2016). L'échec d'une distinction claire entre ces deux domaines - philosophique et clinique - peut générer des malentendus et produire des pratiques cliniques trompeuses (Tatossian \& Samuelian, 2002). En partant d'un dialogue ouvert entre la phénoménologie et la psychiatrie, on peut considérer que plusieurs dénominations peuvent être attribuées à la phénoménologie clinique, comme, par exemple, phénoménologie psychiatrique, psychopathologie phénoménologique et Daseinsanalyse (Charbonneau, 2010), soit en fonction de la proximité épistémologique, soit comme résultat de ce dialogue (Bloc, Moreira, Chamond \& Wolf-Fédida, à paraître). Nous employons ici le terme phénoménologie clinique en raison de son amplitude et de sa capacité à intégrer le champ psy autour de la clinique en se présentant à la fois comme une base pour la rencontre clinique et une source de compréhension phénoménologique du vécu psychopathologique. 
Pour Tatossian (2002/1979, 1986), la relation entre les phénoménologies philosophique et clinique doit être d'implication et non d'application. Cela signifie que l'arrivée de la phénoménologie dans le champ clinique ne doit pas signifier la transposition de la phénoménologie d'un domaine à l'autre, une simple application technique, mais la création d'une base structurelle qui peut permettre un regard spécifique sur l'expérience comme un lieu de questionnement, pour penser phénoménologiquement la clinique et le vécu psychopathologique (Moreira \& Bloc, 2016). Il faut savoir ce que veut dire «être impliqué phénoménologiquement » : il s'agit de l'attitude phénoménologique où le clinicien rencontre le sujet en respectant toujours le propre mouvement de celui-ci tout en visant le dévoilement de l'expérience qui se présente à lui. Pour voir, il faut un mode de voir qui, dans ce cas, peut être fourni par l'implication phénoménologique et c'est ce qui rend possible la «vraie» phénoménologie clinique. D'un côté, la formulation d'une phénoménologie clinique refusant la phénoménologie philosophique comme base constituante, serait une restriction de ses possibilités et, surtout, un non-usage de la fécondité de cette relation, mais d'un autre côté, la simple transposition de la phénoménologie philosophique à la clinique peut être un enjeu dangereux dans la mesure où elle peut ne devenir qu'un discours théorique sur le sujet, loin de la clinique et du contact avec le patient.

Pour permettre la relation entre la phénoménologie clinique et la phénoménologie philosophique, Tatossian propose que le clinicien travaille en flux entre la théorie et la pratique (Moreira \& Bloc, 2016), c'est-à-dire jamais statiquement, mais toujours en établissant une base solide ancrée sur l'expérience. En d'autres termes, le clinicien a besoin de la connaissance descriptive de plusieurs vécus psychopathologiques, que la tradition de la phénoménologie clinique construit depuis son début, par exemple, avec Binswanger et Minkowski, mais il ne devra jamais rester fixé à cette connaissance et au contraire il devra la placer entre guillemets «pour apprendre avec le patient» dans l'expérience clinique. La phénoménologie porte en effet un potentiel clinique en tant qu'école de l'expérience (Tatossian, 2002/1979). Cela veut dire que sa condition d'inachèvement et de (re)composition en fonction du mouvement de chaque patient permet de remettre en question tout l'apport théorique. Il y a toujours un élément du vécu qui peut nous faire apprendre mais pour qu'il y ait apprentissage, il faut une ouverture capable de permettre l'apparition de ce qui est nouveau et propre à l'expérience de chaque patient. 
Être avec un patient signifie, comme le dit Tatossian (2002/1979), être à la fois avec lui, avec son mode d'être-au-monde, et avec sa pathologie qui apporte des éléments communs référents à un vécu psychopathologique. Pour atteindre ce vécu, et mieux le comprendre, la théorie se présente comme un outil, non statique, pour s'approcher de l'autre et de son expérience, pour le saisir. Ce discours se développe de la clinique et pour la clinique (Moreira, 2016), c'est-à-dire que toutes ses sources proviennent de la clinique et contribuent éminemment à «l'être-avec » de l'acte clinique. Il y a vraiment mutuelle constitution entre la théorie et la pratique. Dans la phénoménologie clinique que nous pratiquons, la notion de corps propre est notre outil pour comprendre le vécu et s'approcher de l'expérience du patient.

\section{Le corps propre dans la phénoménologie philosophique de Merleau-Ponty}

La notion de corps propre chez Merleau-Ponty (1992/1945) ne consiste pas qu'en un corps objectivement constitué, un corps physique en tant qu'une machine inerte ou instrument extérieur. Il s'agit du corps vécu dans l'intersection homme et monde qui doit être saisi comme «l'enveloppe vivante de nos actions »(Merleau-Ponty, 2006/1942, p. 206). Dans toute son œuvre, il essaie de surmonter la pensée dualiste corps/âme caractéristique de la science moderne. Ses deux premiers travaux, par exemple, touchent vraiment «au problème traditionnel des relations de l'âme et du corps » (Merleau-Ponty, 2010/1951, p. 1813), c'est-à-dire des rapports qui s'établissent entre le sujet et les conditions organiques de sa vie en mettant l'accent sur la perception. Cette préoccupation lui fait prendre le corps sensible comme centre de sa philosophie, puisque le corps est «le véhicule de l'être-aumonde » (Merleau-Ponty, 1992/1945, p. 97). Ce corps est toujours présent et engagé tout en abandonnant la position d'objet $\mathrm{du}$ monde pour être reconnu comme moyen de communication avec le monde.

L'œuvre Phénoménologie de la perception, où la notion de corps propre est traitée, correspond au début de sa phénoménologie existentielle, axée sur la compréhension de l'homme comme être en situation. Ce corps habituel (vécu) lie le physiologique et le psychique pour une intégration de l'existence, qui se construit à chaque instant. À ce stade de son travail Merleau-Ponty (Merleau-Ponty, 1992/1945) développe le concept de corps propre, le comprenant en tant que médiateur de toute expérience possible. Le philosophe propose une analyse phénoménologique de notre ouverture au monde à travers le corps et fait valoir que le 
corps ne peut pas être considéré comme une chose ou un objet et propose de surmonter la conception objective et mécanique du corps en la remplaçant par l'idée du corps comme sens et signification, ce qui est justement le corps propre (Bloc et al., 2015). En d'autres termes, dans la présentation du corps propre, il doit y avoir quelque chose qui le rend perceptible mais aussi quelque chose qui traduit son absence, car «il n'est ni tangible, ni visible dans la mesure où il est ce qui voit et ce qui touche » (Merleau-Ponty, 1992/1945, p. 108). Notre existence est corporelle comme une « incarnation perpétuelle » (Merleau-Ponty, 1992/1945, p. 194) qui nous met toujours en relation avec le monde. C'est avec le corps, notre corps, que nous sommes au monde, tout à fait ancrés dans l'existence.

Avec un «mode d'exister particulier » (Barbaras, 2005, p. 69) au-delà d'une objectivation, le corps propre est «adhésion active au monde » (Barbaras, 2005, p. 69) : « mon corps est caractérisé par le fait qu'il est toujours de mon côté, avec moi plutôt que devant moi » (Barbaras, 2005, p. 69). Le corps propre est mon corps dans le sens de l'intimité avec le corps que je vis, que je sens et que j'éprouve en tant que mien. Par ailleurs, cela pose le vrai problème du corps propre qui «consiste en ceci que tout y demeure » (Merleau-Ponty, 1992/1945, p. 230). Pour connaître le corps humain, soit le corps d'autrui, soit mon corps propre, il faut le vivre, il n'y a pas d'autre manière, dit Merleau-Ponty.

La relation entre l'homme et le corps est pré-réflexive et ambiguë. Lorsque MerleauPonty (Merleau-Ponty, 1992/1945) affirme « je ne suis pas devant mon corps, je suis dans mon corps, ou plutôt je suis mon corps » (p. 175), il aborde un corps qui est toujours présent pour moi, et en même temps, qui fait partie d'un monde qui l'entoure ; il s'agit d'une coexistence et d'une co-appartenance. Ce corps a un certain style qui ne peut pas être comparé à un objet physique, mais à une œuvre d'art. Comparer le corps à une œuvre d'art, c'est la manière trouvée par Merleau-Ponty (1992/1945) pour dire à la fois que le corps est un nœud de significations et s'éloigner de l'idée du corps en tant qu'objet physique :

Un roman, un poème, un tableau, un morceau de musique sont des individus, c'est-à-dire des êtres où l'on ne peut distinguer l'expression de l'exprimé, dont le sens n'est accessible que par un contact direct et qui rayonnent leur signification sans quitter leur place temporelle et spatiale. C'est en ce sens que notre corps est comparable à l'œuvre d'art. Il est un nœud de significations vivantes et non pas la loi d'un certain nombre de termes covariants (p. 177).

Merleau-Ponty essaie vraiment de surmonter la vision dualiste de la science positiviste en percevant l'homme dans une constitution mutuelle avec le monde, avec 
l'histoire et la culture. Cette perspective imprègne la construction de la notion de corps comme un organisme intégré, comme étant simultanément le sujet et l'objet (Bloc et al., 2015). Le corps propre est à la fois ouverture, comme un « je peux » toujours en nous mettant en situation mais aussi fermeture lors de manifestations pathologiques. Ainsi, ce corps est «un ensemble de significations vécues qui va vers son équilibre » (Merleau-Ponty, 1992/1945, p. 179) dans l'espace de la relation sujet-objet et de l'ambiguïté en tant que touchant-touché, voyant-vu.

Cette idée est illustrée par l'exemple célèbre de Merleau-Ponty: lorsque je mets ensemble mes deux mains, est-ce la main droite qui touche la gauche ou est-ce la main gauche qui touche la droite? En fait les deux sont touchés-touchantes :

quand je touche ma main droite avec ma main gauche, l'objet main droite a cette singulière propriété de sentir, lui aussi. Nous avons vu tout à l'heure que jamais les deux mains ne sont en même temps l'une à l'égard de l'autre touchées et touchantes. Quand je presse mes deux mains l'une contre l'autre, il ne s'agit donc pas de deux sensations que j'éprouverais ensemble, comme on perçoit deux objets juxtaposés, mais d'une organisation ambiguë où les deux mains peuvent alterner dans la fonction de «touchante» et de «touchée » (MerleauPonty, 1992/1945, p. 109)

L'organisation ambiguë permet l'alternance de fonctions apparemment opposées. Le recouvrement entre main touchant et main touchée n'est pas possible. Seul le corps propre permet une telle organisation et une telle ambigüité en produisant une tension, une possible alternance de fonction et de production de sens. Selon Merleau-Ponty (2000/1960), «la distinction du sujet et de l'objet est brouillée dans mon corps » (p. 211). Ainsi, il y a une inversion constante des rôles de sujet et d'objet dans le corps (Barbaras, 2005). La réversibilité du toucher, étape clé en la direction de l'ambigüité, devient imprécise comme toute appropriation dichotomique du corps car tous les essais pour établir une nuance en tant que sujet ou objet ne sont toujours que partiels.

Ainsi l'ambiguïté traverse la notion de corps propre chez Merleau-Ponty. Pour lui, « dire que j'ai un corps c'est une façon de dire que je peux être vu comme un objet et que j'essaie d'être considéré comme sujet » (Merleau-Ponty, 1992/1945, p. 195), c'est-à-dire que ce corps n'est pas seulement un objet, car il est enraciné dans le monde, ainsi que notre existence, et qu'il a à la fois un rôle actif et un rôle passif. Le corps implique notre condition d'être dans le monde et se présente comme la condition de possibilité de l'identité du sujet et 
d'orientation vers le monde (Zielinski, 2002). Il s'agit d'un sujet corporel dans son incarnation dont la caractéristique évoque la condition de coexistence, sans confusion ni séparation, du corps propre et du monde dans leur tissu charnel.

Merleau-Ponty rompt définitivement avec la notion du corps comme un objet dans la mesure où il propose une conception de l'homme impliqué dans le monde et non comme sujet de relations causales, tel que le propose la conception dualiste de l'homme. La discussion clinique en psychopathologie, à partir de l'idée de corps propre, du point de vue de la phénoménologie existentielle présentée par Merleau-Ponty (1992/1945), donne la possibilité de comprendre le vécu du patient au-delà, ou en-deçà, des symptômes, c'est-à-dire, en dépassant l'approche diagnostique traditionnelle, sans toutefois la nier (Bloc et al., 2015). Le grand défi d'une phénoménologie clinique du corps propre est à la fois de se tourner vers le mouvement constant du vécu corporel tout en ne succombant pas à la tentation objectiviste de considérer d'une façon critique les discours sur ce vécu comme partie des significations produites dans le monde et par le monde.

\section{Le corps vécu dans la phénoménologie clinique d'Arthur Tatossian: l'exemple de la dépression}

Inspirée par la phénoménologie philosophique initiée par Husserl et reprise par Heidegger, puis par Merleau-Ponty et Sartre, la phénoménologique clinique, aussi bien que la psychopathologie phénoménologique, a commencé au début des années 1920 avec les études de Ludwig Binswanger et Eugène Minkowski (Tatossian \& Azorin, 1984). Depuis son origine, dans ses différents courants, elle se concentre sur la compréhension du vécu, soulignant généralement la nécessité d'un contact clinique qui permet le retour à l'expérience. Le corps vécu se présente en tant que paradigme de la compréhension de l'expérience et point de vue effectif dans la phénoménologie clinique en étant capable d'atteindre les conditions de possibilité du vécu psychopathologique. En choisissant la dépression, nous illustrons ce regard face à l'ancrage corporel de l'expérience et la prise du corps dans sa dimension intersubjective, sensible et non dichotomique.

Tatossian a publié plusieurs articles importants sur la dépression, qui, pour lui, ne peut se concevoir sans un corps, car, de même que les humains, les animaux eux-mêmes sont une existence incarnée. Cette compréhension se réfère à ce qu'il appelle le corps vécu ou encore corps vivant, différent du corps des anatomistes ou des physiologistes, et où ce qui est 
appelé soma ou psyché ne sont que des abstractions ou des développements secondaires (Tatossian et al., 2016; Tatossian, 2002/1979 ; Tatossian, 1977, 1981, 1982, 2016/1983).

Lorsque Tatossian (1982) parle de la question du corps, il souligne que ce corps est constamment ignoré, étant objectivé, de manière éphémère, dans des circonstances telles que la faim, la soif, la fatigue, entre autres expériences. Il montre le temps, le corps, l'espace et la relation avec l'autre comme des conditions de possibilité du vécu dépressif. Pour comprendre le corps vécu, Tatossian (1982) établit une distinction entre «le corps que je suis et qui ne fait qu'un avec moi et le corps que j'ai et qui m'étant disponible comme instrument et même comme chose, n'est justement pas moi dans un certain sens » (1982, p. 99). Le corps que je suis ou corps-sujet désigne la propre expérience du corps, un corps dans lequel je me reconnais alors que le corps que j'ai ou le corps-objet est différent du soma et agit comme une interface avec le monde, permettant l'intersubjectivité dans un corps qui peut être vu comme l'autre le voit. Ce sont deux pôles nécessaires dont l'équilibre marque un fonctionnement sain (Bloc et al., 2015).

Dans le cas du vécu dépressif, Tatossian (1982, 2016/1983) suggère un éventuel déséquilibre entre le corps-sujet et le corps-objet. Le corps-objet, le corps-que-j'ai disparaît et le déprimé finit pour s'identifier uniquement au corps-sujet. Sans le corps-objet, le sujet perd l'intermédiation avec le monde, il n'y a plus ni point d'appui ni de connexion. Des gestes simples deviennent extrêmement difficiles pour le déprimé car le contact avec le monde n'est plus disponible ou insuffisant. En ce qui concerne le contact vital, dévitalisé dans la dépression, Tatossian (2016/1983) estime qu'il ne s'agit pas du contact émotionnel ou sensoriel, mais plutôt de «la source de l'un et de l'autre, c'est-à-dire de la communication vitale comme capacité de mise en relation avec le monde»(p. 282). Le déprimé vit une absence dont l'origine est « l'effacement du corps-objet, la difficulté ou l'impossibilité d'objectiver son propre corps tout en restant Soi » (2016/1983, p. 282), ce qui lui fait perdre la proximité existentielle avec le monde qui semble être loin, hors de portée.

Merleau-Ponty (1997/1935-1951) a écrit très tôt sur le corps avec lequel nous faisons cause commune, et cela va au-delà d'une simple relation avec le corps, comme présence, car il existe une sorte d'adhérence et d'intimité entre moi et le corps. Dans cette perspective le corps est beaucoup plus que quelque chose que l'on possède, car placé entre ce que je suis et ce que j'ai. Ce n'est plus purement l'objet car il est situé à la frontière entre ce que je suis et ce que j'ai. Il y a là, pour Merleau-Ponty (1997/1935-1951), un mouvement entre être et avoir un 
corps qui caractérise la condition humaine, car «Ce n'est jamais notre corps objectif que nous mouvons, mais notre corps phénoménal (corps vécu) » (p. 153).

On peut dire que cette condition, dans le cas de la dépression, se trouve malmenée, car ce corps qui sert de pont pour la relation avec le monde est «effacé », tout se passe comme si le corps avait été enlevé (Bloc et al. 2015). Comme nous le rappelle Merleau-Ponty (Bloc et al., 2015), «Il faut bien qu'à chaque moment de notre vie nous sachions où est notre corps sans avoir à le chercher comme nous cherchons un objet déplacé pendant notre absence » (p. 156), vu que le corps est expression naturelle et que c'est avec lui que nous comprenons les autres et percevons les choses. Tout demeure dans le corps, c'est lá le problème du monde et du corps propre, dit Merleau-Ponty. La maladie est une «forme d'existence » (Merleau-Ponty, 1992/1945, p. 138) et le corps se montre comme voie d'expression. Le malade «est son corps et son corps est la puissance d'un certain monde » (Merleau-Ponty, 1992/1945, p. 137). Dans le cas de la dépression, vivre ce corps est extrêmement difficile car le mouvement et l'équilibre d'être et avoir un corps n'existe plus le vécu est lourd et vide du fait que le corps en tant qu'objet est effacé. Ce qui était simple, naturel, habituel devient désormais difficile ou presque impossible dans le vécu dépressif.

\section{Conclusion}

L'idée de corps propre présentée dans la phénoménologie philosophique de MerleauPonty permet l'expansion fructueuse de la compréhension et de l'approche du corps dans le domaine de la phénoménologie clinique. Bien qu'il n'explicite pas dans ses écrits la relation directe de ses idées avec la question du corps propre, trouvée à l'origine dans le travail de Merleau-Ponty, Tatossian développe une pensée clinique dans l'espace du corps propre, pourrions-nous dire. Comprendre le corps comme inter-corporéité, comme corps propre, demande un regard clinique éminemment ambigu. Il s'agit de comprendre le corps à la fois comme sujet et comme objet. Autrement dit, il ne s'agit pas seulement d'un corps comme sujet - le corps que je suis, comme le dirait Tatossian mais ce n'est pas non plus le corps objet - le corps que j'ai, tellement à la mode de nos jours.

Le monde contemporain met l'accent sur ce corps objet en oubliant son rôle intersubjectif et essaie de plus en plus de le contrôler. Le corps est souvent vu comme une machine, en série, ce qui conduit à un éloignement du vécu et de son caractère propre. La large gamme des troubles alimentaires, par exemple, est une démonstration de l'importance 
excessive donnée au corps-objet et souligne la dimension pathologique du déséquilibre entre être et avoir un corps.

À travers la notion de corps propre, la phénoménologie clinique, inspirée par et impliquée avec la phénoménologie de Merleau-Ponty, est susceptible d'avoir une contribution importante dans le développement de traitements et d'interventions qui comprennent de façon ambiguë le corps comme sujet et objet, touchant et touché, le corps que je ne peux être que si je l'ai. 


\section{Références}

Angelino, L. (2008). L'apriori du corps chez Merleau-Ponty. Revue Internationale de Philosophie, 244, 167-187.

Barbaras, R. (2005). De la phénoménologie du corps à l'ontologie de la chair. In J.-C. Goddard, Le Corps (pp. 207-250). Paris: Vrin.

Bimbenet, E. (2011). Après Merlaeu-Ponty: Études sur la fécondité d'une pensée. Paris: Vrin.

Bloc, L., Melo, A., Leite, E., \& Moreira, V. (2015). Fenomenologia do corpo vivido na depressão. Revista Estudos em Psicologia (Natal), 20(4), 217-228.

Bloc, L., Moreira V., Chamond, J. \& Wolf-Fédida, M. (no prelo). La relation d'implication (et non d'application) entre les phénoménologies philosophique et clinique: le point de vue d'Arthur Tatossian. Bulletin de Psychologie.

Charbonneau, G. (2010). Introduction à la psychopathologie phénoménologique (Vol. 1). Paris: MJW Fédition.

Merleau-Ponty, M. (2006). La Structure du Comportement. Paris: Presses Universitaires de France. (Oeuvre originale publieé en 1942).

Merleau-Ponty, M. (1997). Parcours. Paris: Éditions Verdier. (CEuvre originale publieé en 1935-1951).

Merleau-Ponty, M. (1992). Phénoménologie de la perception. Paris: Gallimard. (Euvre originale publieé en 1945).

Merleau-Ponty, M. (2000). Signes. Paris: Galimard. (Oeuvre originale publieé en 1960).

Merleau-Ponty, M. (2010). Travaux et projet d'enseignement. In Merleau-Ponty, Euvres. Paris: Gallimard. (Euvre originale publieé en 1951).

Moreira, V. (2016). Partie II. In A. Tatossian, V. Moreira, J. Chamond, J. Tatossian, J. Pita, L. Bloc \& et. al, Clinique du Lebenswelt: psychothérapie et psychopatholgie phénoménologiques (pp. 147-264). Paris: MJW Fédition.

Moreira, V., \& Bloc, L. (2016). Clinique du Lebenswelt (monde vecú): articulation et implication entre thérie et pratique. In A. Tatossian, V. Moreira, \& J. e. Chamond, Clinique du Lebenswelt: psychothérape et psychopathologie phénoménologiques (pp. 253-264). Paris: MJW Fédition.

Roux, S. (2010). Le statut du corps dans la philosophie platonicienne. In J.-C. Goddar, Le Corps (pp. 11-42). Paris, França: Vrin. 
Rovaletti, M. L. (1984). El cuerpo como lenguaje, expresión y comunicación. Revista de Filosofia, XVII(51), 491-504.

Tatossian, A. (1982). A Phénoménologie du corps. In E. Jeddi, Le corps en psychiatrie (pp. 99-103). Paris: Masson.

Tatossian, A. (2016). Dépression, vécu dépressif et orientation thérapeutique. In A. Tatossian, V. Moreira, J. Chamond, J. Tatossian, J. Pita, L. Bloc \& et. al, Clinique du Lebenswelt: psychotérapie et psychopathologie phénoménologiques (pp. 99-116). Paris: MJW Fédition. (CEuvre originale publieé en 1983).

Tatossian, A. (2002). La phénoménologie des psychoses. Paris: Le Cercle Hermeneutique, Collection «Pheno ». (Oeuvre originale publieé en 1979).

Tatossian, A. (1977). Le sens de la dépression. Méditerranée Médical, 5(146), 33-36.

Tatossian, A. (1981). Phénoménologie de la dépression. Enchéphale, 7, 361-366.

Tatossian, A. (1986). Pratique Psychiatrique et Phénoménologie. In P. Fédida, \& P. Gutton, Phénoménologie, Psyquiatrie, Psychanalyse (pp. 123-131). Paris: Echos-centurion.

Tatossian, A., \& Azorin, J.-M. (1984). Phénoménologie. In A. Porot, Mannuel Alphabetique de Psychiatrie (pp. 528-529). Paris: Presses Universitaires de France.

Tatossian, J., \& Samuelian, J-C. (2002). Posface. In A. Tatossian, La phénoménologie des psychoses (pp. 239-244). Paris: Le Cerle Hermeneutique, Collection « Pheno ».

Zielinski, A. (2002). Lecture de Merleau-Ponty et Levinas: Le corps, le monde, l'autre. Paris: Presses Universitaires de France. 